\section{Trastornos prolongados de conciencia postinjuria cerebral aguda}

\author{
FERNANDO VERGARA $^{1}$
}

\section{Prolonged disorders of consciousness after an acute brain injury}

Only a fraction of patients in coma secondary to a primary acute brain injury develop a vegetative state (VS). At least $20 \%$ of patients show late transitions to a minimally conscious states (MCS). They are particularly common in young adults with traumatic brain injury. The main problems faced by clinicians are the diagnostic accuracy of VS and MCS as well as the usefulness of sophisticated paraclinical investigations. Specific therapies are of limited effectiveness. This population is vulnerable to misdiagnosis and limited access to medical care and rehabilitation, thus generating ethical problems.

(Rev Med Chile 2019; 147: 1621-1625)

Key words: Coma; Coma, Post-Head Injury; Persistent Vegetative State.
${ }^{1}$ Clínica Universidad de los Andes. Santiago, Chile.

Trabajo no recibió financiamiento. El autor declara no tener conflictos de interés.

Recibido el 25 de junio de 2019, aceptado el 20 de enero de 2020.

Correspondencia a: Dr. Fernando Vergara Clínica Universidad de los Andes Avenida Plaza 2501. Santiago. fvergarae@gmail.com

\section{Trastornos de conciencia prolongados postcoma}

$\mathrm{E}$ l coma es un estado autolimitado que se resuelve en un período no mayor a cuatro semanas y, de no mediar la muerte o su recuperación, evoluciona con grados variables de compromiso funcional. Después de la introducción de medidas de cuidado intensivo, especialmente de la respiración asistida, un pequeño número de pacientes con injurias agudas cerebrales graves, tales como anoxia o traumatismos encefalocraneanos que en décadas pasadas fallecían, pueden evolucionar a un estado vegetativo o de mínima conciencia.

Un comité constituido por The American Academy of Neurology, The American Congress of Rehabilitation Medicine y The National Institute on Disability, Independent Living and Rehabilitation Research ha publicado recientemente una guía con un riguroso y exhaustivo análisis crítico de la literatura con criterios basados en evidencia sobre el diagnóstico, pronóstico y utilidad de los exámenes paraclínicos ${ }^{1}$. Esta guía excluye estudios con menos de 20 pacientes y los categoriza de acuerdo al nivel de evidencia.
La citada guía también reconoce que la mayor parte de las recomendaciones presenta niveles limitados de evidencia. Estas están basadas en un escaso número de pacientes. Los reportes no son estandarizados y constan de un seguimiento limitado. La guía insiste en la precaución que se debe tener sobre el pronóstico, ya que algunos pacientes que han estado por tiempo prolongado en estado vegetativo pueden mejorar a largo plazo. Por este motivo, se recomienda el término del estado vegetativo crónico.

\section{Nosología de los trastornos de conciencia}

Los trastornos de conciencia secundarios a injurias cerebrales difusas o multifocales incluyen varios síndromes clínicos que se han definido a través de las respuestas conductuales. Es importante precisar que las distintas categorías diagnósticas son sindromáticas, es decir, que corresponden a un fenotipo que tiene distintos mecanismos patogénicos, neuropatológicos y de pronóstico variable. Además tienen, en muchos casos, un perfil evolutivo en el tiempo, dependiendo de la noxa y de las medidas terapéuticas, que incluyen 
la rehabilitación. El pronóstico depende de la fisiopatología de la injuria, la extensión y localización de las lesiones, la edad y las comorbilidades. En su primera etapa los pacientes están en coma, lo que representa un estado de ausencia de vigilancia y de contenidos de conciencia. De no mediar la muerte o su recuperación, después de la primera semana se evidencia una recuperación de la vigilancia con compromiso variable de los contenidos de la conciencia.

Estos trastornos se han clasificados en dos categorías: estado vegetativo (EV) y de mínima conciencia (EMC). Hay que reconocer que ambos estados son parte de un continuum sin límites precisos y que evolucionan en el tiempo. Según la guía, existe un $20 \%$ de transición a EMC por parte de pacientes que cumplían criterios de EV. Esto, especialmente, en sujetos jóvenes con injurias cerebrales traumáticas ${ }^{2}$.

La taxonomía de los estados prolongados de conciencia se inicia con la introducción (Jennett y Plum, 1972) del estado vegetativo (EV) ${ }^{3}$. Posteriormente, se introdujeron los estados de mínima conciencia (EMC) y la emergencia del estado de mínima conciencia (EEMC) $)^{4-6}$.

El estado vegetativo se caracteriza por la emergencia del coma con apertura ocular y aparentes ciclos de la vigilia del sueño. No hay evidencias de experiencias conscientes. Pueden estar presentes respuestas reflejas o espontáneas, tales como el rechinar de dientes, llanto, bostezos, movimientos coreiformes o clonus.

En los estados de conciencia mínima hay evidencias menores, pero evidentes, de conciencia de sí mismos y del medio ambiente. Las respuestas son variables e inconsistentes. Los signos presentes incluyen fijación de la mirada hacia objetos o personas, como también localización de estímulos nociceptivos o manipulación de objetos. En algunos pacientes puede haber algún grado de verbalización inteligible. La clasificación de estados de mínima conciencia en pacientes con lenguaje $(\mathrm{EMC}+)$ y sin lenguaje (EMC-) no implica necesariamente un grado mayor de gravedad o implicancias pronósticas, sino que más bien constituyen el reflejo de la localización y magnitud de las lesiones cerebrales. Durante los últimos años se ha demostrado en pacientes en EV y EMC, especialmente postraumáticos, la presencia de actividad cerebral encubierta ante órdenes, utilizando paradigmas de imaginería visual a través de resonancia magnética y EEG. Se ha propuesto el término de disociación cognitivo motora para describir estos pacientes que son incapaces de expresar conductualmente procesos cognitivos. Tales pacientes, clasificados desde el punto de vista clínico como EV, justificarían el diagnóstico de $\mathrm{EMC}^{7,8}$. Esta subcategoría está basada en datos preliminares. Recientes reportes de estudios con paradigmas activos demuestran que hay un porcentaje alto de falsos negativos y positivos que puede resultar de la aplicación de diferentes métodos estadísticos en este grupo de pacientes ${ }^{9}$. Por este motivo parece recomendable mantener los términos clínicos clásicos en $\mathrm{EV}$, EMC y EEMC, describiendo el estado conductual, con escalas tales como el Coma Recovery Scale ${ }^{10}$. La emergencia del estado de conciencia mínima se caracteriza por un estado delirioso con defectos mnésicos.

Un diagnóstico diferencial importante es el síndrome de enclaustramiento (locked in). Producido por lesiones pontinas ventrales, se caracteriza por cuadriplejia y anartria con conservación de la conciencia. Los pacientes mantienen los movimientos oculares verticales y de párpados, lo que permite la comunicación no verbal. Se caracteriza por un defecto en las eferencias motoras, por lesiones a nivel del puente -con conservación íntegra de la conciencia- y por la ausencia de comunicación, salvo a través de movimientos oculares verticales ${ }^{5}$.

\section{Diagnóstico}

Los trastornos prolongados de conciencia deben cumplir con criterios: una duración de al menos cuatro semanas, ser causado por una injuria cerebral de causa conocida, que sea compatible con el trastorno prolongado de conciencia y que el coma no sea secundario a causas médicas. El diagnóstico sigue siendo clínico a través de una evaluación neuroconductual. La precisión diagnóstica mejora significativamente cuando se usan escalas estandarizadas Una de las más utilizadas es la Coma Recovery Scale-Revised ${ }^{10}$. Es recomendable evaluar a los pacientes de forma seriada y en distintos momentos del día, dadas las fluctuaciones normales de conciencia

Se ha mencionado que hay un error de diagnóstico de hasta $40 \%$ en pacientes calificados de estados vegetativos que corresponderían a 
EMC $^{11}$. Esto no debe llevar a la conclusión de que los pacientes estén despiertos o en estado de enclaustramiento, cuya incidencia es muy baja. Sin embargo, esta frecuencia pudiera ser exagerada ya que está basada en un número reducido de pacientes con reportes subóptimos y con seguimiento insuficiente. También hay que considerar la transición de un estado a otro en el curso del tiempo. Curiosamente, no hay reportes de error de diagnóstico en pacientes con estados de mínima conciencia, cuando se trata más bien de un estado vegetativo. Es importante mencionar que aquellos pacientes que han evolucionado desde un EV y de mínima conciencia muestran defectos cognitivos de magnitud variable, no alcanzando una cognición normal ${ }^{12}$.

La evaluación neurológica debe establecer que el tálamo y la corteza hayan sufrido un daño severo, excluyendo un daño primario del tronco cerebral sin compromiso talámico o hemisférico. La evaluación del estado de conciencia debe tomar en consideración otras condiciones médicas reversibles, tales como la epilepsia y el uso de drogas sedantes. Otro factor de confusión es la presencia de afasia, defectos visuales y compromiso motor y sensitivo.
El diagnóstico diferencial preciso es importante ya que el EMC diagnosticado en los primeros meses tiene un importante grado de recuperación funcional a los 12 meses, en comparación con el EV.

\section{Procedimientos diagnósticos paraclínicos}

La utilidad de paraclínicos es aún limitada en la práctica. Ninguno de los procedimientos paraclínicos presenta sensibilidad ni especificidad adecuadas. Los estudios electrofisiológicos, incluyendo respuesta EMG a comandos motores, reactividad del EEG, potenciales evocados e índice de perturbación compleja inducida por TMS (estimulación transcraneal magnética), tienen un valor limitado en la distinción entre EMC y EV. La obtención de las neuroimágenes funcionales en la identificación de la actividad cognitiva residual es metodológicamente compleja, de alto costo y sólo está disponible en centros de alta tecnología. La ausencia de activación cerebral no descarta una mejoría futura. No hay evidencias suficientes para refutar o apoyar el uso clínico de rutina de imágenes funcionales (fMRI o PET) ${ }^{1}$.

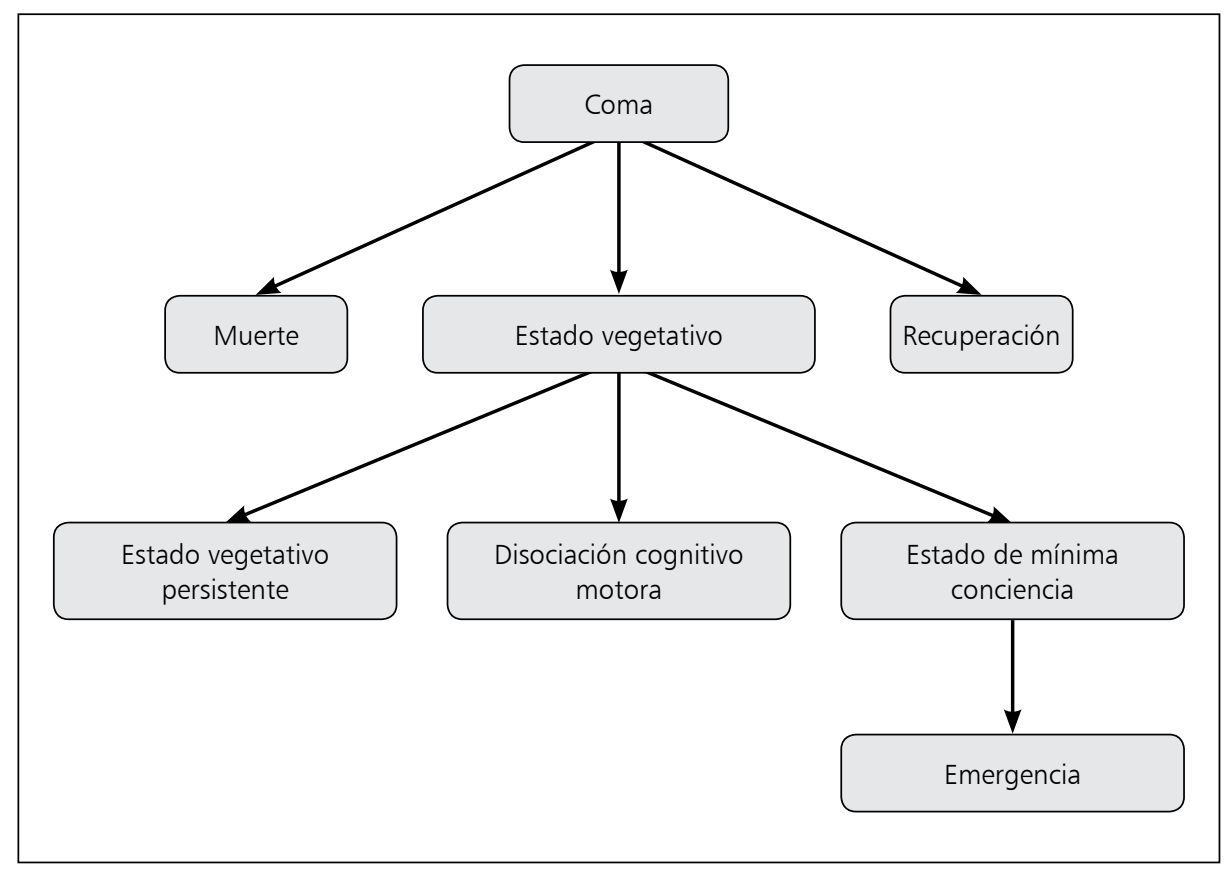

Figura 1. Evolución del coma. 


\section{Pronóstico}

La incidencia de estos estados es de 2,6/10.000/ año y la prevalencia de entre 2,0 a $5 / 100.000^{1}$. La mayoría de los pacientes se mantienen en este estado en forma prolongada. Una minoría sustancial, de hasta $14 \%$ a $20 \%$ de los EV, tiene una transición a EMC, especialmente en pacientes jóvenes con injurias traumáticas. Aun cuando los pacientes presentan discapacidad severa, algunos recuperan la capacidad de interacción social y comunicación. Se deben evitar afirmaciones de un mal pronóstico durante los primeros 28 días por parte de los tratantes.

En los EV de causa no traumática, la mayor parte de los pacientes permanecerán en este estado después de tres meses. El 17\% de ellos puede emerger a un EMC a los 6 meses; y 7,5\%, después de 24 meses. En los EV traumáticos, 38\% emerge a los 3 meses; y el 78\%, a los 12 meses. Aun cuando con un grado de discapacidad importante, $20 \%$ de los pacientes postinjuria traumática recupera algún grado de capacidad funcional a los cinco años. Los pacientes con diagnóstico de EMC, en los primeros cinco meses después de la injuria tienen un mejor pronóstico funcional que aquellos en $\mathrm{EV}$.

Las últimas recomendaciones han preferido usar el término de estado vegetativo crónico, ya que el término permanente implica irreversibilidad. Esto, basado en que un porcentaje de pacientes sometidos a tratamiento médico y rehabilitación intrahospitalaria, especialmente en los casos postraumáticos, pueden evolucionar a una independencia funcional, posiblemente por reinervación de circuitos corticales ${ }^{1}$.

\section{Tratamiento}

En la etapa aguda del coma es difícil establecer un pronóstico en las primeras $72 \mathrm{~h}$, en parte por la dificultad de determinar el estado neurológico, ya sea por la presencia de fármacos sedantes, hipotermia o condiciones médicas concomitantes. No parece prudente el retiro de las medidas de apoyo en esta etapa, incluso cuando esto es frecuente en pacientes con injuria cerebral grave en casos de etiologías traumáticas, especialmente no traumáticas, durante las primeras $72 \mathrm{~h}$ desde la admisión.

Una situación especial es la de discutir la opción de no intentar resucitar al paciente, comu- nicando a la familia que la ocurrencia de un paro cardiorrespiratorio agravaría el daño preexistente.

Posteriormente, se debe revisar con la familia la limitación del esfuerzo terapéutico, especialmente en pacientes con daño anóxico isquémico. Esta decisión puede estar determinada por los deseos previos del paciente o de los familiares, pero también por el pronóstico del equipo médico. Así, parece prudente mantener las medidas terapéuticas por un plazo de al menos 28 días ${ }^{13}$.

La mayor parte de los reportes de tratamiento de los estados crónicos de conciencia son de validez limitada, con un número escaso de pacientes y sin estudios prospectivos. La mayor parte de los estudios fueron excluidos, ya que se hicieron en la etapa aguda, sin grupos de control y, por lo mismo, resultan metodológicamente incorrectos. No hay evidencia suficiente sobre la eficacia de tratamientos tales como la cámara hiperbárica, estimulación cerebral profunda o estimulación vagal, uso de amantadina, levodopa, bromocriptina o zolpidem. Existe un estudio randomizado del uso de amantadina por cuatro semanas en pacientes postraumáticos en casos de $\mathrm{EV}$ o de EMC, el cual demostró una mejoría funcional en comparación al grupo placebo. La guía clínica sugiere que pacientes en casos EV o MC, entre las cuatro y 16 semanas, deben recibir amantadina de 100 a $200 \mathrm{mg}$, dos veces al día, para permitir la recuperación funcional ${ }^{1}$. Evidencias limitadas de eficacia se han demostrado por estimulación transcraneal magnética ${ }^{1,14}$. El control adecuado del dolor puede requerir medicación que afecte la cognición. Por este motivo parece recomendable minimizar los fármacos sedantes en la etapa aguda. Una escala recientemente introducida, llamada Nociception Coma Scale Revised), es útil para identificar la presencia de dolor ${ }^{15}$.

\section{Consideraciones éticas y paliativas}

Con la evidencia disponible es importante evitar predicciones categóricas de pronóstico funcional en pacientes que sobreviven al primer mes. Considerando las dificultades diagnósticas y la posibilidad de evolución de estados vegetativos crónicos, se hace difícil limitar las medidas terapéuticas de sostén, especialmente en los casos secundarios a traumatismos encefalocraneanos. En la etapa aguda son esenciales el control de las 
complicaciones médicas, el control del dolor y la neurorrehabilitación. Es necesario comunicar de forma adecuada la incertidumbre pronóstica, ya que ésta es probabilística.

Debe, asimismo, explorarse la limitación del tratamiento de condiciones médicas intercurrentes, tales como cáncer, infecciones e insuficiencia renal, en los plazos pronósticos del estado vegetativo prolongado.

En los EV prolongados, una consideración importante es la suspensión de las medidas de sostén, especialmente de alimentación. Es fundamental incorporar en la discusión con los familiares los deseos previos del paciente cuando esto es constatado. Por el contrario, es sumamente complejo cuando no existen instrucciones previas y hay desacuerdo entre los cuidadores.

\section{Referencias}

1. Giacino JT, Katz DI, Schiff ND, Whyte J, Ashman EJ, Ashwal S, et al. Practice guideline update recommendations summary: disorders of consciousness. Neurology 2018; 91 (10): 450-60.

2. Estraneo A, Trojano L. Prognosis in Disorders of Consciousness. In: Schnakers C, Laureys S. (eds) Coma and Disorders of Consciousness. Editorial Springer. 2018. p 17-36.

3. Jennett B, Plum F. Persistent vegetative state after brain damage: a syndrome in search of a name. Lancet 1972; 299 (7753): 734-7.

4. Giacino JT, Ashwal S, Childs N, Cranford R, Jennett B, Katz DI, et al. The minimally conscious state: Definition and diagnostic criteria Neurology 2002; 58 (3): 349-53.

5. Posner JB, Saper CB, Schiff ND, Claassen J. Plum and Posner's Diagnosis and Treatment of Stupor and Coma (Contemporary Neurology Series) 5th Edition Editorial
Oxford 2019 p. 398-405.

6. Wijdicks EFM, Cranford RE. Clinical diagnosis of prolonged states of impaired consciousness in adults. Mayo Clin Proc 2005; 80 (8): 1037-46.

7. Bruno MA, Vanhaudenhuyse A, Thibaut A, Moonen G, Laureys S. From unresponsive wakefulness to minimally conscious PLUS and functional locked-in syndromes: recent advances in our understanding of disorders of consciousness. J Neuro 2011; 258 (7): 1373-84.

8. Schiff ND. Cognitive motor dissociation following severe brain injuries. JAMA Neurol 2015; 72 (12): 1413-5.

9. Peterson A, Cruse D, Naci L, Weijer C, Owen AM. Risk, diagnostic error, and the clinical science of consciousness. Neuroimage Clin 2015; 7: 588-97.

10. Giacino J, Kalmar K, Whyte J. The JFK Coma Recovery Scale-Revised: measurement characteristics and diagnostic utility. Arch Phys Med Rehabil 2004, 85 (12): 2020-9.

11. Schnakers C, Vanhaudenhuyse A, Giacino J, Ventura M, Boly M, Majerus S, et al. Diagnostic accuracy of the vegetative and minimally conscious state: clinical consensus versus standardized neurobehavioral assessment. BMC Neurol 2009; 9 (35): 35-5.

12. Wade DT. How often is the diagnosis of the permanent vegetative state incorrect? A review of the evidence. Eur J Neurol 2018; 25 (4): 619-25.

13. Dale CM, Sinuff T, Morrison LJ, Golan E, Scales DC. Understanding Early Decisions to Withdraw Life-Sustaining Therapy in Cardiac Arrest Survivors. Ann Am Thorac Soc 2016; 13 (7): 1115-22.

14. Thibaut A, Schiff N, Giacino J, Laureys S, Gosseries O. Therapeutic interventions in patients with prolonged disorders of consciousness. Lancet Neurol 2019; 18 (6): 600-14.

15. Schnakers C, Chatelle C, Vanhaudenhuyse A, Majerus S, Ledoux D, Boly M, et al. The Nociception Coma Scale: a new tool to assess nociception in disorders of consciousness. Pain 2010; 148 (2): 215-9. 